\title{
Impaired CCR7 Expression on Plasmacytoid Dendritic Cells in HIV Infected Children on HAART With Virologic Failure Seema Desai* ${ }^{* 1}$, Aida Chaparro ${ }^{2}$, Huanliang Liu ${ }^{1}$, Patrick Haslett ${ }^{1}$, Gwendolyn Scott ${ }^{2}$, Rajendra Pahwa ${ }^{3}$ and Savita Pahwa ${ }^{1}$
}

Address: ${ }^{1}$ Department of Microbiology and Immunology, University of Miami Miller School of Medicine, Miami, FL 33136, USA, ${ }^{2}$ Department of Pediatrics, University of Miami Miller School of Medicine, Miami, FL 33136, USA and ${ }^{3}$ Diabetes Research Institute, University of Miami Miller School of Medicine, Miami, FL 33136, USA

Email: Seema Desai* - SDesai@med.miami.edu

* Corresponding author ¥Presenting author

from 2005 International Meeting of The Institute of Human Virology Baltimore, USA, 29 August - 2 September 2005

Published: 8 December 2005

Retrovirology 2005, 2(Suppl I):P28 doi:I0.I I86/I742-4690-2-SI-P28

\section{Background}

Defects of myeloid (m) DC and plasmacytoid (p) DC are well established in HIV infection. Studies in children and adolescents are limited, and have focused mainly on IFN$\alpha$ function of pDCs.

\section{Materials and methods}

Patients with perinatal HIV infection ( $\mathrm{n}=19$, ages 11-18 $\mathrm{yr}$, on HAART) were classified as immunologic responders (IR+; CD4>25\%), and virologic responders (VR; plasma HIV RNA < 400 copies $/ \mathrm{mL}$ ). mDC (Lin-, HLA $\mathrm{DR}+\mathrm{CD} 11 \mathrm{c}+$ ) and pDC (Lin-, HLA DR+ CD11c-) were evaluated in a novel whole blood assay by flow cytometry for expression of maturation markers CD83, CD80, homing receptor CCR7 and intracellular cytokines (TNF- $\alpha$ and IFN- $\alpha$ ) after short-term stimulation with a TLR7/8 agonist, resiquimod.

\section{Results}

CCR7 expression was markedly reduced in pDC of IR-VRsubjects in comparison to IRVR patients (mean $5.6 \%$ vs $43.3 \%$ ). Levels of CCR7 were intermediate (mean 29.8\%) in the IR+VR- group, and were almost absent in two patients with VL>100,000 copies who had a markedly reduced IFN- $\alpha$ production in pDC. CD83, CD80, and TNF-a were expressed in all patients and were more pronounced in $\mathrm{mDC}$ than in $\mathrm{pDC}$.

\section{Conclusion}

The most striking finding was a reduced expression of CCR7, and is indicative of a defect in homing of the plas- macytoid DC to lymph nodes in HIV infected children who have ongoing active viral replication and poor immunologic control in spite of HAART. 